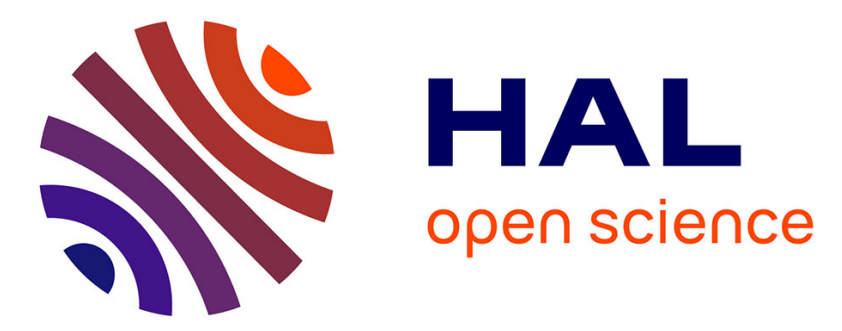

\title{
High temperatures and Raman scattering through pulsed spectroscopy and CCD detection
}

\author{
P. Simon, B. Moulin, E. Buixaderas, N. Raimboux, E. Hérault, B. Chazallon, \\ H. Cattey, N. Magneron, J. Oswalt, D. Hocrelle
}

\section{- To cite this version:}

P. Simon, B. Moulin, E. Buixaderas, N. Raimboux, E. Hérault, et al.. High temperatures and Raman scattering through pulsed spectroscopy and CCD detection. Journal of Raman Spectroscopy, 2003, 34 (7-8), pp.497-504. 10.1002/jrs.1020 . hal-01996753

\section{HAL Id: hal-01996753 \\ https://hal.science/hal-01996753}

Submitted on 27 Sep 2021

HAL is a multi-disciplinary open access archive for the deposit and dissemination of scientific research documents, whether they are published or not. The documents may come from teaching and research institutions in France or abroad, or from public or private research centers.
L'archive ouverte pluridisciplinaire HAL, est destinée au dépôt et à la diffusion de documents scientifiques de niveau recherche, publiés ou non, émanant des établissements d'enseignement et de recherche français ou étrangers, des laboratoires publics ou privés. 


\title{
High temperatures and Raman scattering through pulsed spectroscopy and CCD detection
}

\author{
P. Simon, ${ }^{1 *}$ B. Moulin, ${ }^{1 \dagger}$ E. Buixaderas, ${ }^{1}$ N. Raimboux, ${ }^{1}$ E. Herault, ${ }^{2 * *}$ B. Chazallon, ${ }^{1 \ddagger}$ \\ H. Cattey, ${ }^{1 \S}$ N. Magneron, ${ }^{19}$ J. Oswalt ${ }^{3}$ and D. Hocrelle ${ }^{3}$ \\ ${ }^{1}$ Centre de Recherches sur les Matériaux à Haute Température, CNRS, F-45071 Orléans Cedex 2, France \\ 2 ESPEO-IPO, 12 rue de Blois, Université d'Orléans, BP 6744, F-45067 Orléans Cedex 2, France \\ 3 Jobin-Yvon, 16-18 rue du Canal, F-91195 Longjumeau Cedex, France
}

\begin{abstract}
A Raman scattering system devoted to high temperatures is presented. It is based on a pulsed system to remove thermal emission with retaining charge-coupled device (CCD) detection. Two types of optical gating are used: a Pockels switch or an intensified CCD (ICCD), combining in this case the optical gate and the CCD detection. The performances of both systems are presented and their respective advantages are discussed and compared. The ICCD device allows recording of spectra in the $2000^{\circ} \mathrm{C}$ range, and typical results obtained on alumina and zirconia ceramics are shown. Copyright $\odot 2003$ John Wiley \& Sons, Ltd.
\end{abstract}

KEYWORDS: Raman scattering; Pockels; high temperature; zirconia; intensified charge-coupled device

\section{INTRODUCTION}

Raman scattering is widely used in numerous applications in materials science, from the laboratory level up to online control in industrial facilities. The peculiarities of the method allow complex and severe sample environments, through optical fibers and delocalization of the laser and spectrometer. Nevertheless, high-temperature applications remain non-routine measurements, at least for temperatures exceeding $1000^{\circ} \mathrm{C}$. The term 'high temperature' in the present paper means values above this arbitrary limit. This field corresponds to the temperature of elaboration, thermal treatment or melting of many very common materials, mainly oxides such as refractories, silicas and glasses, and earth science compositions (aluminates, silicates, etc.).

This somewhat arbitrary value of $1000^{\circ} \mathrm{C}$ corresponds in fact to some limit below which Raman scattering, with classical continuous exciting radiation in the green or blue range,

*Correspondence to: P. Simon, Centre de Recherches sur les Matériaux à Haute Température, CNRS, F-45071 Orléans Cedex 2, France. E-mail: simon@cnrs-orleans.fr

Contract/grant sponsor: National Agency for Valorization (ANVAR).

${ }^{\dagger}$ Present address: LMN, Université Evry Val d'Essonne, F-91025

Evry Cedex, France.

$\ddagger$ Present address: PhLAM, USTL, F-59655 Villeneuve d'Ascq Cedex, France.

§Present address: LSEO, Université Bourgogne, 6 Bld Gabriel, F-21000 Dijon, France.

Il Present address: IUFM, F-45044 Orléans Cedex 1, France.

**Present address: Institut d'Optique UMR 8501, CNRS Bat 503,

Centre Universitaire d'Orsay F-91403 Orsay, France. does not exhibit specific difficulties. At higher temperatures, recording a Raman spectrum will become more and more difficult owing to the thermal emission originating from the hot sample, which will rapidly become much more intense and will completely mask the vibrational information. Although several authors have reported successful Raman measurements at these extreme temperatures (briefly reviewed in the next section), these experiments could not be considered routine measurements and were performed with detection systems largely less powerful than charge-coupled devices (CCDs) which offer today an invaluable level of performance. It was then of interest to try to conceive a Raman device with CCD detection, devoted to high temperatures. The experience acquired at CRMHT on spectroscopic measurements at high temperature (x-ray scattering ${ }^{1}$ and EXAFS, ${ }^{2} \mathrm{NMR}^{3}$ and particularly spectral emissivity ${ }^{4}$ ) is an important advantage for this challenge, and on the other hand, as high-temperature materials are mostly frequently strongly disordered, it is then important to characterize them with different spectroscopic methods in order to have information at different length and time scales.

Different approaches are possible to limit the effect of thermal emission on Raman measurements. They are reviewed in the next section and it appears that time resolved methods are the most efficient. Two different experimental devices for pulsed Raman spectroscopy with CCD detection have been developed: the first is based on an ICCD (intensified CCD) replacing the classical CCD detector and the second is a Pockels switch positioned on the scattered 
beam, between the sample and the entrance slit of the spectrometer.

The present paper is organized as follows: the next section is devoted to the fundamentals of thermal emission and to a review of the different ways to access Raman information in high-temperature conditions, then the details of the experimental set-up are given and the final section reports some examples of high temperature Raman data obtained with these devices.

\section{THERMAL EMISSION AND RAMAN SCATTERING}

Any material at non-zero temperature emits an electromagnetic spectrum whose intensity and wavenumber depend on temperature, the direction of emission and the material itself. The ideal blackbody absorbs all the incident radiations and its spectral luminance is given by the well-known Planck's law:

$$
L_{0}(\sigma, T)=\frac{C_{1} \sigma^{3}}{\mathrm{e}^{\frac{C_{2} \sigma}{T}}-1}
$$

$\left(C_{1}=1.191 \times 10^{-16} \mathrm{~W} \mathrm{~m}^{-2}, C_{2}=1.439 \mathrm{~m} \mathrm{~K}\right)$ where $\sigma$ is the wavenumber and $T$ the absolute temperature. In the visible spectral range, which is the most common one for Raman devices, the blackbody luminance becomes noticeable around $1000^{\circ} \mathrm{C}$, where it corresponds to typical intensities of Raman scattering in current oxides. Of course, as Raman intensities are very strongly dependent on the material and on the experimental set-up, this temperature is not a precise threshold. Nevertheless, owing to the exponential dependence on $T$, this limit will not move by several hundred degrees.

Any real material has a luminance $L(\sigma, T, \theta)$ lower than the blackbody material ( $\theta$ is the angle of incidence) and can be characterized by its spectral emissivity $\varepsilon$ :

$$
\varepsilon(\sigma, T, \theta)=\frac{L(\sigma, T, \theta)}{L_{0}(\sigma, T)}
$$

This shows that to limit the luminance $L$ and to overcome the difficulty of measuring Raman scattering in a real sample, namely scattering in a hot sample, two approaches are possible: either limiting the contribution of the blackbody term $L_{0}$, or limiting the emissivity. This latter point is frequently forgotten and is the first of the different means of accessing Raman spectra in high-temperature conditions to be developed.

\section{Controlling sample emissivity}

Equation (2) shows the important role of the material itself on the thermal flux which is emitted. In fact, as quoted by Kirchoff's first law, the spectral emissivity is equal to the spectral absorptivity. Then, in a spectral range where there is no absorption mechanism, there will be negligible emission. An easy verification of this is to compare two silica samples, one chemically highly pure and the other containing $3 \mathrm{~d}$ ion impurities: if they are heated near their $T_{\mathrm{g}}\left(1200^{\circ} \mathrm{C}\right)$ the first one appears to the eye to the practically as room temperature, whereas the second will be strongly emitting in the orange-yellow range. Then, to improve Raman detection, it is important to limit all the absorption mechanisms, if possible. In addition, the quality of the surfaces, and also the volume, are important points: any scattering mechanism increases the travel of the light inside the sample and hence increases the effective absorption of the light.

It is then better to work on optically polished and optically transparent (in the absolute Raman spectrum range) materials, when available. Of course, this is not always possible. Characterization of ceramics at high temperatures, for example, is of high interest; further, some specific types of disorder and hence of scattering and/or absorption will become more and more important at high temperatures and they are even the goal of many studies: ionic diffusion and conductivity, softening and ionic motions connected with glass transition, or melting and liquid phases are the main examples of highly interesting systems where it is not possible to limit absorption mechanisms.

\section{Shifting the laser line to the UV range}

The basic idea, underlined over 20 years ago by Farrow and Nagelberg, ${ }^{5}$ is simple: by shifting the excitation laser line to the UV, the Raman spectral range will be further from the maximum of the thermal emission spectrum and hence less contamined by it. This was done in recent years, for instance by Yashima et al. ${ }^{6}$ and Fujimori et al. ${ }^{7}$ by using a UV argon-ion laser radiation $(363.8 \mathrm{~nm})$ for studying zirconia ceramics up to $1500^{\circ} \mathrm{C}$ and hafnia ceramics up to $1700^{\circ} \mathrm{C}$. Zouboulis et al. ${ }^{8}$ performed measurements at even higher energies on a quadrupled YAG: $\mathrm{Nd}^{3+}$ line $(266 \mathrm{~nm})$ with sapphire as sample up to $1450{ }^{\circ} \mathrm{C}$. In both cases the results were satisfactory, and allowed the thermal emission to be rejected sufficiently in the considered temperature range.

As emphasized in both of the above studies, UV Raman spectroscopy is not simple to perform and the experiments were not at all routine. However, now, with the availability of new commercial UV-devoted Raman spectrometers, the situation is different and such high temperatures can be handled more easily. It remains that UV excitation can induce luminescence lines more easily than the visible, and that it is difficult to conceive a spectrometer efficient in both the visible and UV ranges, at least as far as the gratings and their dispersion are concerned. Moreover, most often, the penetration depth is smaller with UV excitation leading to a surface analysis.

If the sample and its surroundings (sample holder, crucible, furnace) act as a blackbody, the solution is then trivially to shift the excitation radiation to higher wavenumber. However, it must be emphasized that the emissivity spectrum is never rigorously equal to 1 at any wavenumber and that, depending of the chemical 
composition, one can have 'holes' in the emissivity spectrum. Therefore, it is then interesting to position the absolute range of the Raman spectrum in one of these holes to limit thermal emission. This is, of course, highly sample dependent, and needs a good knowledge of the emissivity spectrum. It also needs an available laser line in the adapted spectral range. Hence this cannot be a general solution for Raman spectroscopy at high temperatures but it can be useful in some particular cases.

\section{Spatial filtering}

This heading means optical ways to limit the thermal emission recorded by the spectrometer to only the source volume for Raman scattering. Thermal transfers (conductivity, convection or radiative exchanges) lead to a hot region in the sample which is much larger than the volume where the Raman laser is focused. The recorded signal is the sum of the Raman spectrum of a small volume and the thermal signal of a much larger volume. It is then interesting to limit the size of the sample surface which is imaged on the entrance slit of the spectrometer. This can be simply done by positioning two convergent lenses in the parallel beam between sample and entrance slit, and a pinhole localized at the common focus point of the two lenses. This is exactly what is done on a confocal microscope. This advantage of spatial filtering was underlined by Gillet and co-workers ${ }^{9,10}$ (using a confocal microscope) and was also used on the above-cited UV device by Yashima et al. ${ }^{6}$ In the latter case, the experimental device was a macro-Raman type where a supplementary focus point is added for spatial filtering between sample and spectrometer.

\section{Temporal filtering}

The basic idea here is to use the fact that Raman scattering is a very rapid phenomenon, whereas thermal emission is intrinsically continuous. Then, using a pulsed laser and a detection device synchronized with the laser can be a way to discriminate between Raman information and thermal emission. This was reported first by Exharos et al. ${ }^{11}$ and allowed them to record Raman spectra of silica up to $2000^{\circ} \mathrm{C}$. They used for Raman excitation a doubled YAG: $\mathrm{Nd}^{3+}$ laser $(532 \mathrm{~nm})$ and an intensified photodiode array detector.

Later, pulsed Raman spectroscopy was intensively used and developed in Lucazeau's group to monitor in situ the plasma-assisted growth of diamond films. ${ }^{12-15}$ The temperature was lower $\left(1300^{\circ} \mathrm{C}\right)$ than in Exharos et al.'s work but the plasma process adds an intense source of visible light which also needs to be rejected. Excitation and detection devices were of the same type that Exharos et al. used.

Recently, You et al. ${ }^{16}$ reported high-temperature Raman data obtained on a pulsed device, based on a pulsed copper vapor laser and a monochannel time-resolved photomultiplier detector. They obtained remarkable results on sodium disilicate up $1773 \mathrm{~K}$, substantially above its melting-point.
The temporal filtering is represented schematically with ideal square-like pulses in Fig. 1 The pulses of the detection device are synchronized with the laser pulses. Therefore, if $t_{1}$ denotes the pulse length and $t_{2}$ the time between two pulses, the thermal emission is reduced by a rejection factor $r=t_{2} / t_{1}$. This value, depending on the laser and the performance of the detection device, can be of the order of $10^{4}$, with commercial devices (see the Experimental section). Knowing this order of magnitude, it is then relevant to compare what can be achieved concerning the limit temperature in Raman measurements. Figure 2 displays the temperature dependence of the spectral luminance of the blackbody calculated by Planck's law, for $\lambda=532 \mathrm{~nm}$ (wavelength of the doubled YAG: $\mathrm{Nd}^{3+}$ laser), normalized to the value at $1200^{\circ} \mathrm{C}$. This temperature is some limit to classical Raman measurements on a transparent monocrystal. Then, the ordinate in Fig. 2 gives a direct estimation of the rejection factor $r$ which is needed when using a pulsed source at $532 \mathrm{~nm}$. One sees that a value $r \approx 100$ allows to access to temperatures up to $1700^{\circ} \mathrm{C}$, and $r \approx 1000$ to temperatures up to $2200^{\circ} \mathrm{C}$. Figure 3 compares the spectral luminances (assuming a blackbody) at the wavelengths of a doubled and tripled YAG: $\mathrm{Nd}^{3+}$ source; the ratio doubled/quadrupled is also shown for comparison. This figure shows that for a rejection factor of 100, a pulsed system at $532 \mathrm{~nm}$ is more

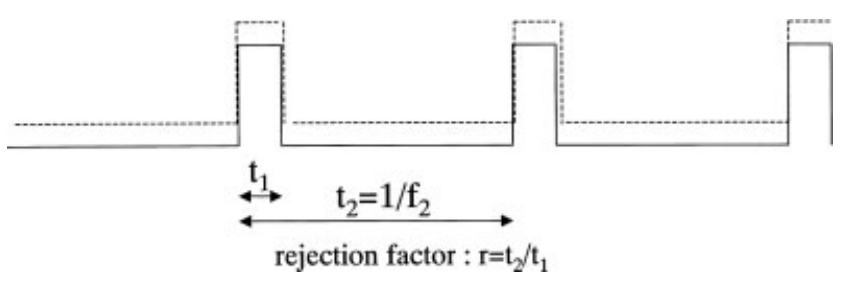

Figure 1. Schematic view of temporal filtering: the continuous line denotes the laser pulses and the dashed line corresponds to the ideal opening times of the detecting device.

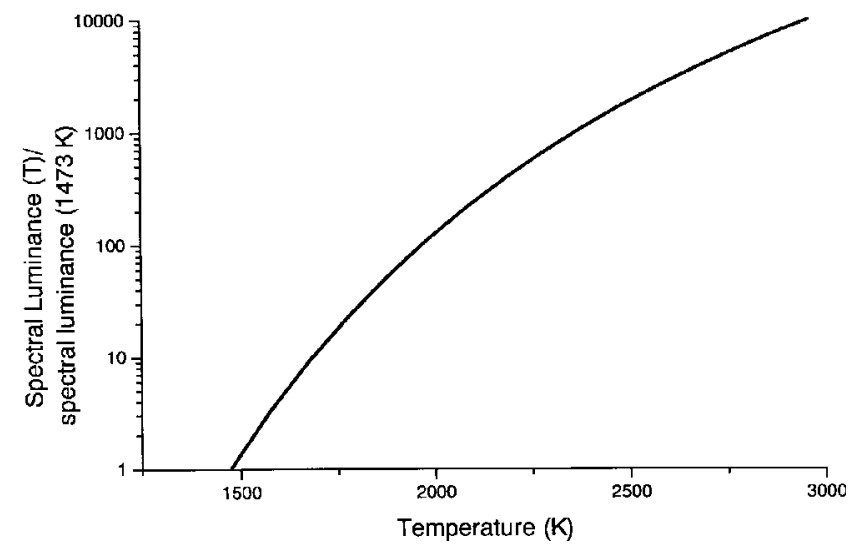

Figure 2. Temperature dependence of the spectral luminance of the blackbody (Planck's law), normalized to the spectral luminance at $1200^{\circ} \mathrm{C}$, at the wavelength $532 \mathrm{~nm}$ (doubled YAG : $\mathrm{Nd}^{3+}$ laser). 


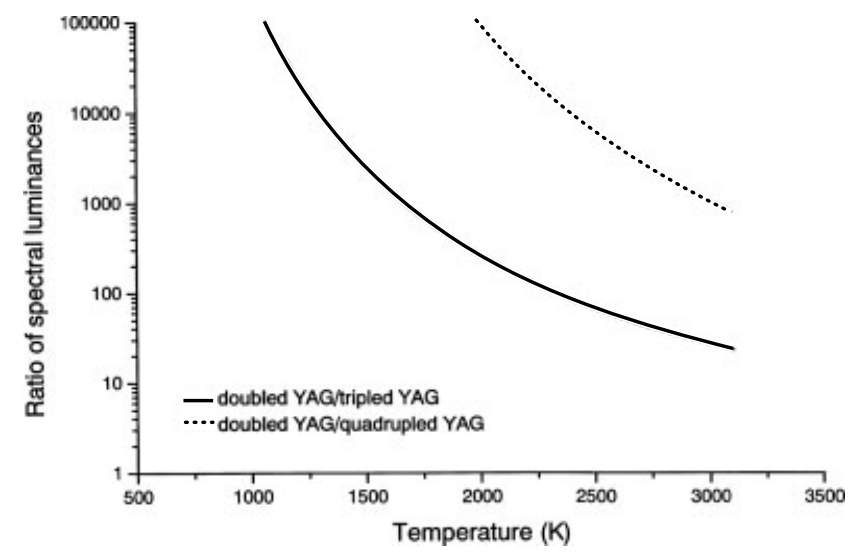

Figure 3. Temperature dependence of the ratios of the spectral luminances of blackbody at different wavelengths. Continuous line, 532/355 nm (doubled YAG/tripled YAG); dotted line, 532/266 nm (doubled YAG/quadrupled YAG).

favorable than a continuous one at $355 \mathrm{~nm}$ above about $2000^{\circ} \mathrm{C}$. This threshold temperature is lowered if the rejection factor is higher. This then leads to the conclusion that a nearUV (355 nm) range is well suited only for 'not too high' temperatures. A continuous quadrupled YAG: $\mathrm{Nd}^{3+}$ source is better, but as seen in Fig. 3, a pulsed $532 \mathrm{~nm}$ source with a rejection factor of $10^{4}$, which is realistic, is even better for temperatures above $2000^{\circ} \mathrm{C}$.

One can postulate combination of both pulsed and UV shift, approaches, which is not unreasonable from the instrumental point of view. The main difficulty in this case will be to control the flux density when focused on the sample, as the damage threshold is attained or even exceeded.

This pulsed approach to high temperature Raman scattering appears to be the most efficient, especially for temperatures of $2000^{\circ} \mathrm{C}$ or above. If compared with classical continuous Raman spectroscopy, the detection device seems to be the weak part of such a system, as either intensified photodiode arrays or photomultipliers have much lower quantum efficiency than CCD devices which today offer invaluable performances. Nevertheless, they looked difficult to integrate in a pulsed Raman system. The aim of the present paper is to report a new experimental Raman device devoted to high temperatures, combining a pulsed system and CCD detection.

In addition to all the methods reviewed here, there are also two well-known and very simple supplementary ways to limit the thermal emission signal which can be used. They are less efficient and hence more interesting when combined with another system, but can be a way to limit the 'leak of thermal emission' following UV excitation or temporal filtering. (i) It is more interesting to record antiStokes rather than Stokes, spectra as obviously the thermal emission is lower (nevertheless, the interest will be lower in the UV range, as the Raman spectrum is shortened to a small absolute wavenumber range). (ii) The old way to record a background spectrum without a Raman laser and to subtract it from the Raman spectrum with a laser can be always useful, even if the acquisition time is doubled.

\section{EXPERIMENTAL}

\section{Excitation laser}

In order to increase the rejection factor $r$, one must decrease the pulse length $t_{1}$ and increase the period $t_{2}$. Besides, if keeping a constant average power, a short $t_{1}$ will lead to too high power densities and sample damage, especially in micro configurations. The laser was then a doubled YAG: $\mathrm{Nd}^{3+}$ (Spectra-Physics FCBar T40-X30-532Q) emitting at $532 \mathrm{~nm}$, with a pulse width of about $40-50$ ns. The shape and width of the pulse are systematically monitored on a scope through a rapid photodiode collecting a small part of the beam at the laser output. The repetition rate used for high-temperature measurements was of the order of $1 \mathrm{kHz}$. The theoretical rejection factor is then about 20000-25000. The energy per pulse at the surface sample is of the order of $50-100 \mu \mathrm{J}$, and one order of magnitude lower when working under a microscope.

\section{Spectrometer}

The spectrometer was a Jobin-Yvon T64000 triple spectrometer in subtractive configuration and multichannel detection, with 1800 grooves $\mathrm{mm}^{-1}$ gratings. It is equipped with macro and micro (Olympus BX40) configurations, both in backscattering. The T64000 macro chamber was kept apart from the spectrometer body by $30 \mathrm{~cm}$ to integrate the components described below.

\section{Pockels switch}

The first device installed to discriminate thermal emission is a Pockels switch, installed on the parallel beam between the sample and the spectrometer entrance slit. It consists of a Pockels cell [Lasermetrics 5046SC-Q1059P ( $\mathrm{KD}_{2} \mathrm{PO}_{4}$ crystal)] placed between crossed polarizers. Without an electric field, the Pockels crystal has its optical axis oriented along the beam and hence leaves the light unchanged. Under an electric field, the crystal becomes birefringent and then modifies the polarization state. For the so-called half-wave voltage ( $3200 \mathrm{~V}$ at $532 \mathrm{~nm}$ ), the crystal is equivalent to a half-wave plate with axes at $45^{\circ}$ from the polarizer direction and then induces a $90^{\circ}$ rotation of the polarization beam. The whole switch is equivalent to crossed polarizers with no transmitted light without voltage, and transmits completely the beam selected by the entrance polarizer upon half-wave voltage. With $90^{\circ}$ rotation of both polarizers and $45^{\circ}$ rotation of a quartz half-wave plate between the Pockels switch and the entrance slit, one can easily record VV or VH configuration spectra, keeping a constant polarization orientation at the spectrometer entrance. The interest of this device is that the commutation time is very rapid, in the nanosecond range. 
In fact, the main limitation of this commutation time is not the Pockels cell itself but its electric supply, limiting the opening-closing cycles to about 40-50 ns.

Another point to verify for the present application is the transmission coefficient of the Pockels switch with absolute wavelength. For a given voltage, the transmission will be equal to 1 (if neglecting the losses inside the crystal and the polarizers) only for one wavelength, for which the crystal is exactly a half-wave plate. The wavenumber dependence of the transmission coefficient is easy to calculate from classical laws of the Pockels effect: ${ }^{17}$

$$
\frac{I_{t}}{I_{0}}(\sigma)=\sin ^{2}\left(\frac{\pi}{2} \frac{\sigma}{\sigma_{\pi}}\right)
$$

where $\sigma_{\pi}$ is the wavenumber of the doubled Nd:YAG laser corresponding to an exactly half-wave shift and $I_{0}$ the incident intensity. A Raman spectrum covers typically $1000 \mathrm{~cm}^{-1}$ and then the maximum loss on this range is of the order of $0.2 \%$. In practice, it is more consistent to adjust the Pockels voltage in order to obtain a half-wave behavior in the middle of the Raman spectral range and not on the laser line.

The Pockels switch can be used over a wide spectral range as needed in Raman scattering. One must underline here that the squared sine transmission curve given in Eqn (3) is valid only for a perfectly parallel beam, exactly aligned along the optical axis of the Pockels crystal, and for ideal polarizers of infinite extinction ratios. All these conditions are, of course, only approached in a real experiment.

This can be improved to some extent by incorporating a beam reducer positioned just before the Pockels cell. It

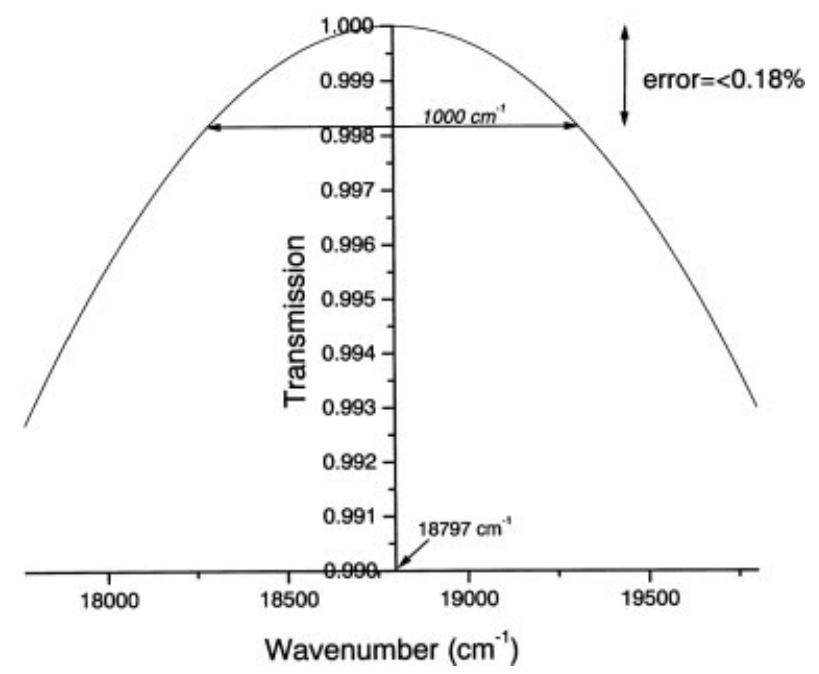

Figure 4. Wavenumber dependence of the transmission coefficient of the Pockels switch for an applied voltage corresponding to half-wave behavior for a doubled $\mathrm{Nd}^{3+}$ : YAG laser $\left(532 \mathrm{~nm}\right.$, i.e. $\left.18797 \mathrm{~cm}^{-1}\right)$. A bandwidth of $1000 \mathrm{~cm}^{-1}$, typical of a Raman spectrum, corresponds to a maximum loss of $0.2 \%$. consists of two lenses of different focal lengths, mounted afocally, with a pinhole at its intermediate focus point where the focal points of both lenses are superposed. The beam is then more parallel, and further the beam diameter is reduced to the size of the Pockels cell. A second identical system (without pinhole) is mounted symmetrically between the Pockels cell and collimating optics on the entrance slit, in order to maintain the nominal aperture of the system.

In this case of a Pockels switch, the detector is the conventional $\mathrm{LN}_{2}$-cooled CCD camera of the Raman spectrometer.

\section{Intensified CCD}

Intensified CCDs (ICCDs) have been available for some years. In these devices, the intensifier is driven by a high voltage allowing transmission and magnification of the signal from the incident photocathode to the CCD chip surface. In the absence of this high voltage, the CCD does not receive any signal. The commutation time can be very rapid, down to a few nanoseconds, and then it is compatible for Raman applications. Therefore, as for the Pockels cell, a high voltage is used to open or close the device. The ICCD used here is a Jobin-Yvon Spectrum One, replacing the classical $\mathrm{LN}_{2}$-cooled CCD camera.

A spatial filter with two lenses and a pinhole (see above) is positioned between the sample and the entrance slit to increase the rejection of thermal emission.

In both Pockels switch and ICCD configurations, the highvoltage pulse is driven by a TTL pulse and delay generator, triggered by the electric command of the Q-switch of the Nd:YAG cavity. This configuration was chosen because the corresponding jitter is lower than in the case where the pulse generator drives both the intensifier and the laser. The delay between the Q-switch trig signal and the opening of the detector is probed in each experiment, as it is strongly dependent on the laser conditions (power and repetition rate). The width of the detector pulse is $50 \mathrm{~ns}$, i.e. the length of the laser pulse. It was confirmed that longer opening times do not lead to more intense signals, as all the Raman information is simultaneous to the laser pulse.

\section{Heating tools}

Two devices were used. For the micro-Raman configuration, a platinum furnace (Linkam TS1500) allows temperatures up to $1400^{\circ} \mathrm{C}$. The sample is put on a monocrystalline $\mathrm{MgO}$ disk inside the furnace; $\mathrm{MgO}$ was chosen owing to its stability upon heating, its absence of first-order Raman modes and for its very low level of impurities giving rise to luminescence.

For the macro configuration and higher temperatures, the heating device is a $150 \mathrm{~W} \mathrm{CO}_{2}$ laser (Coherent Diamond $\mathrm{K}-150$ ). The sample is sphere-shaped (about $1 \mathrm{~mm}$ diameter) and sustained on a gas flow, in a levitation device shown in Fig. 5. This system allows one to work in contactless conditions, which is particularly important when the sample is molten (absence of crucible-liquid interface and hence of chemical reactions at this interface, limitation 


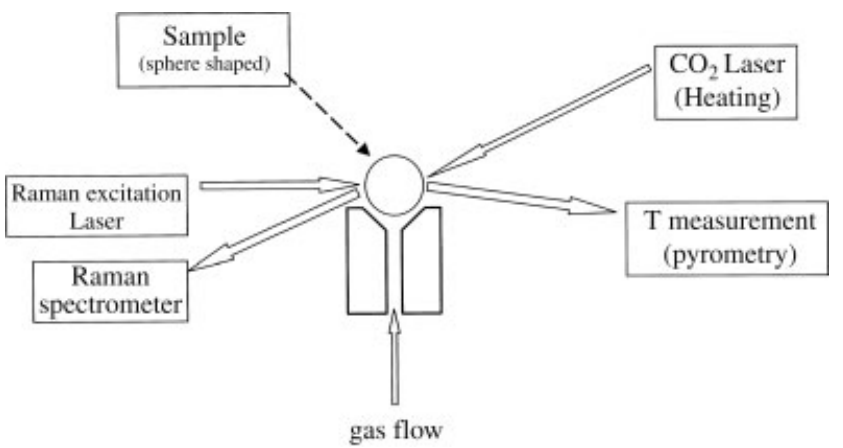

Figure 5. Aerodynamic levitation device for high-temperature Raman measurements in contactless conditions.

of heterogeneous nucleation upon cooling at the liquid-solid transition) and is now incorporated in many spectroscopic or structural characterization devices, e.g. EXAFS, ${ }^{2} x-$ ray $^{1}$ and neutron ${ }^{18}$ scattering and NMR. ${ }^{3}$

\section{APPLICATION TO MODEL MATERIALS}

These experimental tools were tested on model oxides of great interest in the field of high temperatures: alumina and zirconia. Alumina was used either as ceramic rods $(1 \mathrm{~mm}$ diameter) or as spheres; the zirconia samples were always spheres. In all cases the spheres were polycrystalline.

Figure 6 displays the Raman spectra of an alumina ceramic rod, heated by a $\mathrm{CO}_{2}$ laser $\left(1100^{\circ} \mathrm{C}\right)$ with the Pockels switch device. The lowest curve is recorded with the Pockels switch opened, i.e. parallel polarizers and zero voltage to the crystal. The increase to Raman high wavenumbers (low absolute wavenumbers) is just the thermal emission. The second curve is taken with the Pockels switch triggered by the laser: perpendicular polarizers and half-wave voltage applied during the laser pulses. This spectrum is recorded with an acquisition time 100 times longer than the previous one: $10 \mathrm{~s}$ vs $0.1 \mathrm{~s}$. One sees that the thermal emission is reduced by almost two orders of

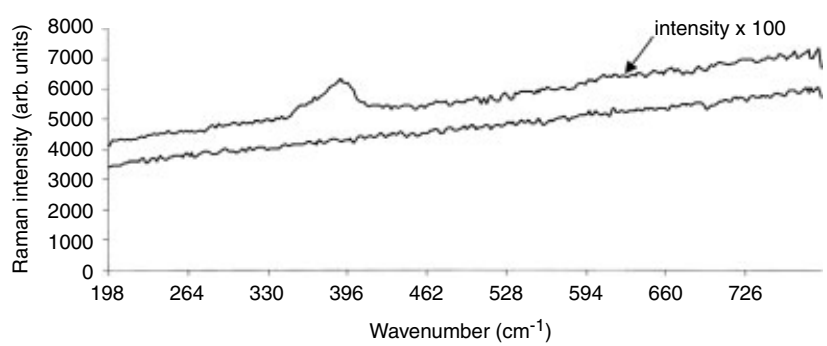

Figure 6. Raman spectra of $\mathrm{Al}_{2} \mathrm{O}_{3}$ ceramics, in macro configuration, with a $\mathrm{CO}_{2}$ laser, recorded with the Pockels switch device. The lower curve was recorded with parallel polarizers and no voltage on the Pockels cell (acquisition time $0.1 \mathrm{~s})$. The upper curve was recorded with perpendicular polarizers and the Pockels cell synchronized on the laser pulse (acquisition time $10 \mathrm{~s}, 100$ times longer than the previous one). magnitude, and the Raman modes near $400 \mathrm{~cm}^{-1}$ become observable. Nevertheless, the baseline is always increasing on going to relative wavenumbers, showing that thermal emission is not completely removed. The rejection of thermal emission is in fact almost two orders of magnitude, far below the theoretical rejection factor of 20000 (see above). In this experiment, no spatial filtering was in place so that the thermal emission was strong because coming from a much larger volume than the Raman information. A spatial filter improves the rejection factor by one order of magnitude. It not only allows one to reject the thermal emission from the sample regions out of the Raman laser spot, but also improves the beam parallelism inside the Pockels switch and its efficiency. The difference from the theoretical rejection factor (one order of magnitude) is explained by a non-ideal alignment of the optical components (Pockels cell, lenses and pinhole) and also by the opening-closing cycles of the Pockels cell which are not so square-shaped as drawn in Fig. 1.

Figure 7 displays similar Raman spectra of alumina ceramics, but obtained this time in a micro configuration with the ICCD device, the sample being heated in the Linkam furnace. It was necessary in this experiment to work with low laser powers, because of the high instantaneous energy density when focused through a microscope. It appears that with the ICCD the rejection of the thermal emission is better, as the baselines are practically flat.

Figure 8 displays Raman results for zirconia ceramics. Figure 8(a) corresponds to the Pockels system and macro configuration; the sample is in aerodynamic levitation. In this case a spatial filter was added to the system between the sample and the Pockels cell (see special filtering section). One sees that now the baselines are flat and that the rejection is better. The highest temperature curve $\left(1400^{\circ} \mathrm{C}\right)$ is noisy, because then the levitation conditions are less stable, presumably owing to macroscopic (facet-shaped) changes on the sample surface after the monoclinic tetragonal transition

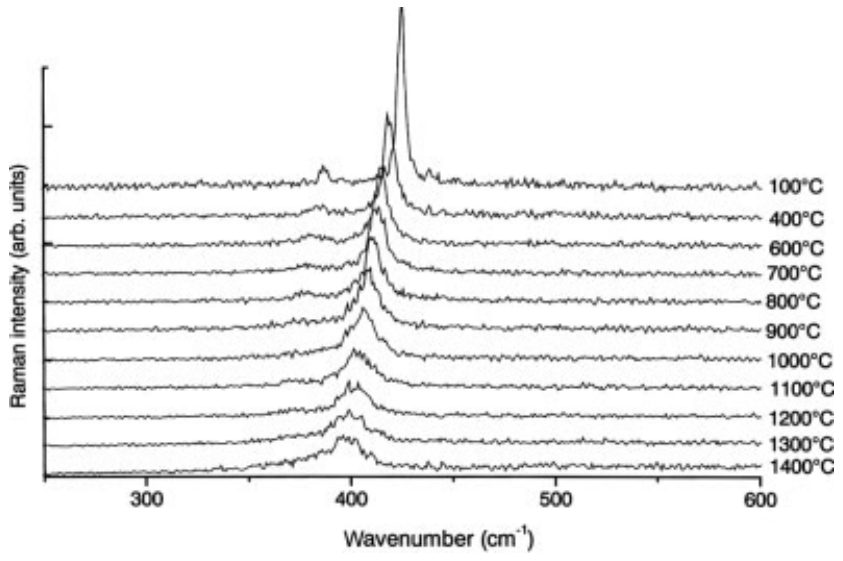

Figure 7. Raman spectra of $\mathrm{Al}_{2} \mathrm{O}_{3}$ ceramics, in micro configuration (heating in furnace), recorded with the ICCD device. Acquisition time, $10 \mathrm{~s}$. 
(a)

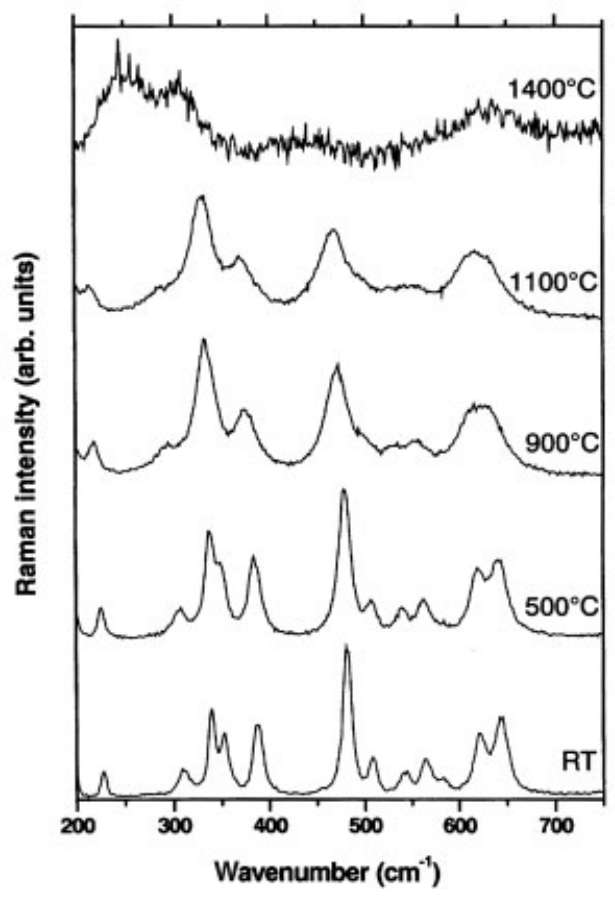

(b)

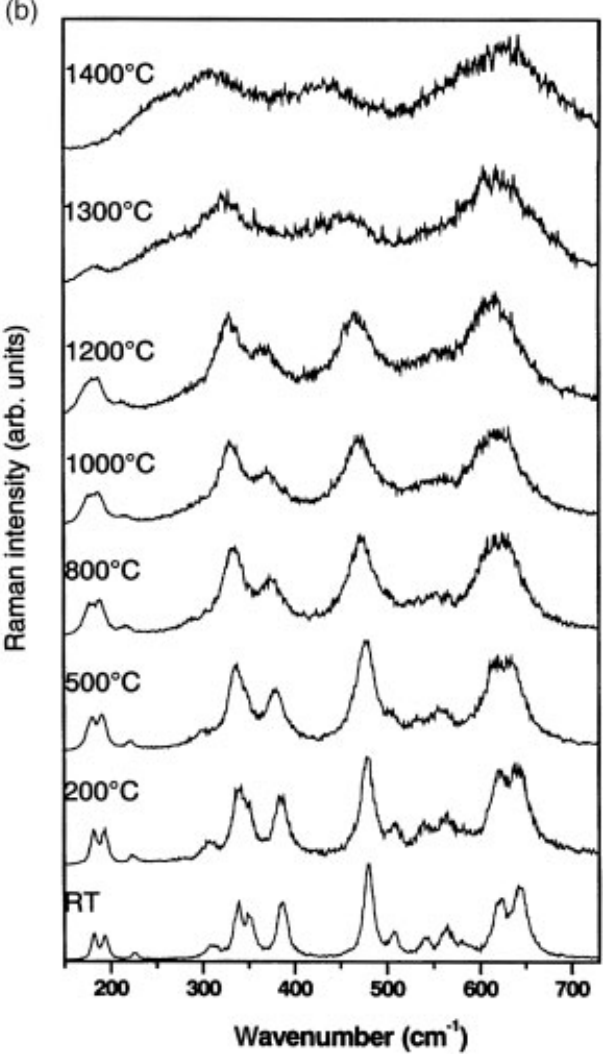

Figure 8. Raman spectra of $\mathrm{ZrO}_{2}$ ceramics through the monoclinic-quadratic phase transition, recorded with (a) the Pockels switch (macro configuration, acquisition time $2.5 \mathrm{~s}$, spatial filtering) and (b) the ICCD (micro configuration, acquisition time $5 \mathrm{~s}$ ).

$\left(1200^{\circ} \mathrm{C}\right)$. The energy per pulse on the sample is about $100 \mu \mathrm{J}$ per pulse, leading, with a spot size of $0.1 \times 0.1 \mathrm{~mm}$, to an average power density of $10 \mathrm{~W} \mathrm{~mm}^{-2}$. Figure $8(\mathrm{~b})$ relates to the micro configuration with the ICCD device and a longer acquisition time ( $5 \mathrm{~s}$ vs $2.5 \mathrm{~s}$ ) in order to limit the energy flux on the sample. The pulse energy is about $10 \mu \mathrm{J}$, so the average power density is estimated to be $400 \mathrm{~W} \mathrm{~mm}^{-2}$ and the spot size is about $5 \times 5 \mu \mathrm{m}$ in this case.

One sees that the spectra look very similar; in the ICCD case the sample is not in aerodynamic levitation and the tetragonal phase spectra $\left(1300\right.$ and $\left.1400^{\circ} \mathrm{C}\right)$ do not appear noisier than the others.

These Raman spectra look similar to those in other work previously reported for the tetragonal phase: You et al., ${ }^{16}$ at high temperature, but also near room temperature, on nanometric zirconia, ${ }^{19}$ on epitaxial films ${ }^{20}$ and by rigid ions model calculations. ${ }^{21}$ Contrary to these last three references, our main goal is not, at least at present, the whole interpretation of the phonon spectrum of tetragonal zirconia, but to define the best way to access it at high temperatures, where the tetragonal phase of macroscopic samples is the equilibrium one.

At this stage it appears that both approaches (Pockels switch and ICCD) are efficient tools for Raman spectroscopy up to $1500{ }^{\circ} \mathrm{C}$. attempts to go to higher temperatures have rapidly shown that the Pockels switch device must be improved (more precise optical alignment, efficiency of the polarizers), which is now in progress. It remains that the main interest of the Pockels device is that it does not add any source of noise to the measurement. Further, the ICCD method looks more powerful today and, to probe its high-temperature limit, in the macro configuration and with laser heating, we have added a spatial filter to increase the rejection of thermal emission. Measurements were made on zirconia and typical results are displayed on Fig. 9. One sees the transition from the tetragonal phase Raman spectrum to a simpler spectrum, dominated by broad lines. The temperature measurement in this case cannot be precise with our experimental setup in its present state, but the temperature is clearly well above $2000^{\circ} \mathrm{C}$. This broad spectrum is the Raman spectrum of the cubic phase of zirconia, above the phase transition near $2300^{\circ} \mathrm{C}$. This is, to our knowledge, the first Raman report on the high-temperature cubic equilibrium phase on pure zirconia. Obviously the linewidths are very broad owing to anharmonicity at these temperatures, but also because of oxygen mobility. The goal is now to improve the experimental conditions at these very high temperatures, in order to improve the detectivity. Then it will be possible to characterize the mechanism of the phase transition and to probe a supposed soft-mode behavior. ${ }^{20}$ It is clear that 


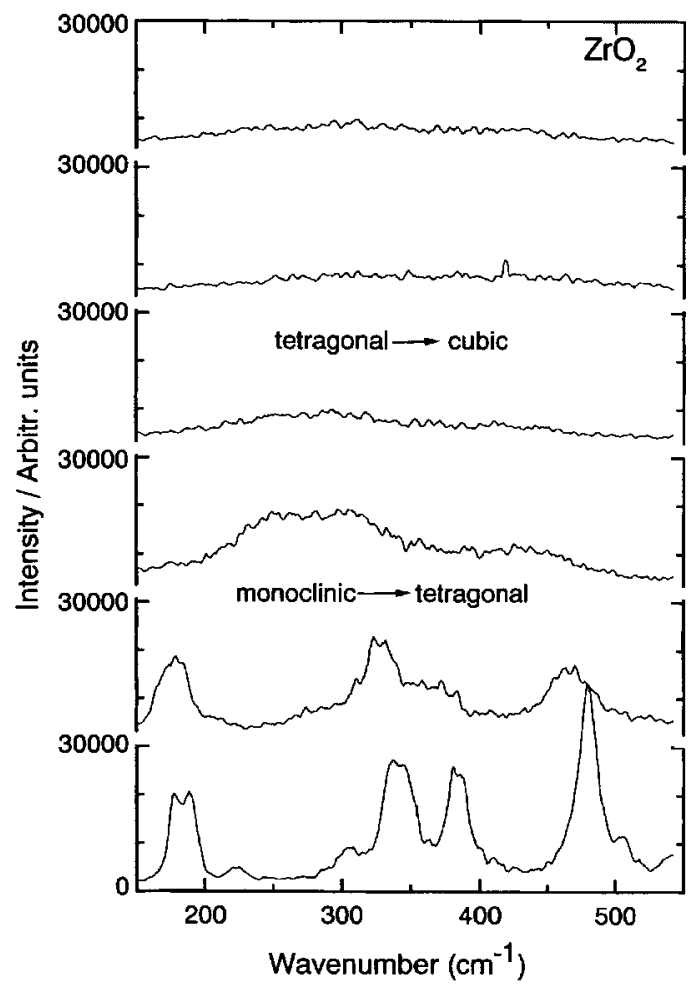

Figure 9. Raman spectra of $\mathrm{ZrO}_{2}$ ceramics (ICCD detection, macro configuration and aerodynamic levitation), showing transition from tetragonal to cubic phase $\left(T_{\mathrm{c}} \approx 2300^{\circ} \mathrm{C}\right)$. Acquisition time, $1 \mathrm{~s}$.

pulsed Raman spectroscopy allows access to temperatures well above $2000^{\circ} \mathrm{C}$, opening the way to many possible fields of investigations, including the liquid phase of refractory oxides.

\section{CONCLUSIONS}

Pulsed Raman spectroscopy with CCD detection was used for probing materials at very high temperatures. Two methods of time-resolved detection were used, a Pockels switch and an ICCD system. Both approaches give access to high temperatures by removing a large part of the thermal emission. The Pockels switch is interesting because it does not add any source of noise to the measurement; however, its performance today is lower than that of the ICCD. The ICCD allows one to reject in a highly satisfactory way the thermal emission, up to more than $2300^{\circ} \mathrm{C}$ in the case of zirconia. Nevertheless, the noise level is slightly higher than for a classical (without intensifier) $\mathrm{LN}_{2}$-cooled CCD. This allows access of Raman scattering spectroscopy to very high temperatures, with interesting possibilities in the characterization of solid phases or even liquid phases, and also in the study of phase transition and glass transition mechanisms. In addition to this high-temperature field, the experimental device is also an easy way to eliminate luminescence components which have characteristic times much longer than the Raman effect. Other approaches, based on a Kerr cell, have recently been developed for time-resolved resonance Raman spectroscopy. ${ }^{22}$

\section{Acknowledgments}

We thank the French National Agency for Valorization (ANVAR) for supporting this work through an Aide à l'Innovation. Thanks are also due to I. Hacala, S. Busson, S. Rager and T. Gibert for their help in developing the Pockels switch device. We also thank Le Studium (Orléans) for a research fellowship for one of us (E.B.).

\section{REFERENCES}

1. Hennet L, Thiaudière D, Gailhanou M, Landron C, Coutures JP, Price DL. Rev. Sci. Instrum. 2002; 73: 124.

2. Landron C, Hennet L, Coutures JP, Gailhanou M, Gramond M, Berar JF. Europhys. Lett. 1998; 44: 429.

3. Massiot D, Trumeau D, Touzo B, Farnan I, Rifflet JC, Douy A, Coutures JP. J. Phys. Chem. 1995; 99: 16455.

4. Rozenbaum O, De Sousa Meneses D, Auger Y, Chermanne S, Echegut P. Rev. Sci. Instrum. 1999; 70: 4020.

5. Farrow RL, Nagelberg AS. App. Phys. Lett. 1980; 36: 945.

6. Yashima M, Kakihana M, Shimidzu R, Fujimori H, Yoshimura M. Appl. Spectrosc. 1997; 51: 1224.

7. Fujimori H, Yashima M, Kakihana M, Yoshimura M. J. Am. Ceram. Soc 2001; 84: 663.

8. Zouboulis E, Renush D, Grimsditch M. Appl. Phys. Lett. 1998; 72: 1.

9. Gillet P. Phys. Chem. Miner. 1996; 23: 263, and references cited therein.

10. McMillan PF, Poe BT, Gillet P, Reynard B. Geochim. Cosmochim. Acta 1994; 58: 3653.

11. Exharos GJ, Frydrych WS, Walrafen GE, Fisher M, Pugh E, Garofalini SH. In Proceedings of the 11th International Conference on Raman Spectroscopy Clark RJH, Long DA (eds). Wiley: New York, 1988; 503.

12. Fayette L, Marcus B, Mermoux M, Rosman N, Abello L, Lucazeau G. J. Appl. Phys. 1994; 76: 1604.

13. Rosman N, Abello L, Chabert JP, Verven G, Lucazeau G. J. Appl. Phys. 1995; 78: 519.

14. Mermoux M, Fayette L, Marcus B, Rosman N, Abello L, Lucazeau G. Analusis 1995; 23: 325.

15. Fayette L, Marcus B, Mermoux M, Rosman N, Abello L, Lucazeau G. J. Mater. Res. 1997; 12: 2686.

16. You J-L, Jiang G-C, Kuangdi X. J. Non-Cryst. Solids 2001; 282: 125, and references cited therein.

17. Huard S. Polarisation de la Lumière. Masson: Paris, 1994

18. Landron C, Hennet L, Jenkins T, Greaves GN, Coutures JP, Soper A. Phys. Rev. Lett. 2001; 86: 4839.

19. Bouvier P, Godlewski J, Lucazeau G. J. Nucl. Mater. 2002; 300: 118; Bouvier P, Lucazeau G. J. Phys. Chem. Solids 2000; 61: 569.

20. Merle T, Guinebretière R, Mirgorodsky A, Quintard P. Phys. Rev. B 2002; 65: 144302 .

21. Mirgorodsky A, Smirnov M, Quintard P. J. Phys. Chem. Solids 1999; 60: 985; Bouvier P, Gupta HC, Lucazeau G. J. Phys. Chem. Solids 2001; 62: 873 .

22. News. Spectrosc. Eur. 2002; 14(4): 8. 